\title{
A developmental study of tactual discrimination in blind and sighted children and adults'
}

ANHE D. PICK AND HERBERT L. PICK, JR. UNIVERSITY OF MINNESOTA

Normally sighted, partially sighted and totally blind Ss from 6 years to adults performed a tactual discrimination task requiring them to judge whether two raised line figures were alike or different. The number of errors made in the task depended on the age of $S$, the amount of vision present, and the nature of the differences between members of the pairs. These results were compared to data obtained by other investigators on visual discrimination of similar figures.

The present study is part of a larger investigation of the relation between the development of visual and tactual perception. A specific visual discrimination task developed by Gibson, Gibson, Pick, \& Osser (1962) was adapted for study tactually and the performance of blind and sighted Ss of different ages was investigated. These results were compared with the visual normative data obtained previously.

Method

A sample of the stimulus forms is shown in Fig. 1. The entire set consists of 12 standard forms and five types of transformation of each: line to curve changes, rotations and reversals, perspective transformations, size changes, and breaks and closes. The forms are replicas of those constructed by Gibson et al (1962) except for the size transformations which were added. For the present study the forms consisted of raised metal lines on a smooth metal background. The forms themselves were approximately 1 in. square.

The Ss were 288 normally sighted individuals, 48 at each of ages $6,7,8,9,11,13$ years; 37 partially sighted (legally blind) Ss, 7-17 years; and 37 totally blind Ss, 7-17 years of age. Both groups of blind Ss had been blind from birth or early infancy and were Braille readers. The partially sighted Ss were able to see at least some movement. The blind Ss were students in a state school for the blind or in special education programs in public schools.

The S's task was to feel a standard form and a comparison form simultaneously, one with each hand, and to judge whether they were exactly the same or different. The forms were presented side by side with the standard form on the left and its comparison on the right. Each $S$ made such judgments on three series of pairs of forms. Each series consisted of a standard form and all the transformations of that form plus one to four copies of the standard. The order of transformations and copies was randomized for each S. The particular standard forms judged by each $S$ were randomized within a group of Ss with the restriction that each standard form was judged the same number

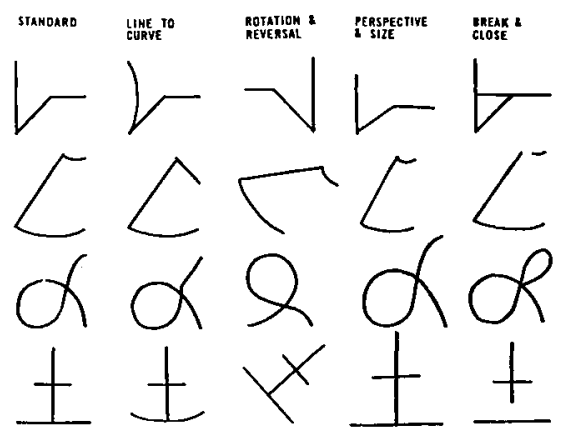

Fig. 1. Examples of standard forms and transformations.

of times. A pretraining procedure was carried out to ensure that all Ss understood the nature of the task and the type of discrimination to be made.

Results

The results are presented graphically in Figs. 2, 3, and 4 in terms of percent confusion errors $a_{0}$ a function of age for each type of transformation. (Size discrimination errors were similar to perspective discrimination errors and are combined with them in these graphs.) For the sighted Ss, the suggestion in Fig. 2 that errors in general decrease with age and that the different types of transformation differ in confusability is confirmed by an analysis of variance showing significant age effects $(F=7.84, \mathrm{df}=5 / 282, \mathrm{p}<.001)$ and transformation effects $(F=461.26, d f=3 / 846, p<.001)$. There was also a significant interaction between age and transformation type $(\mathrm{F}=4.98, \mathrm{df}=15 / 846, \mathrm{p}<.001)$ due to significant age differences for all transformations but perspective and size.

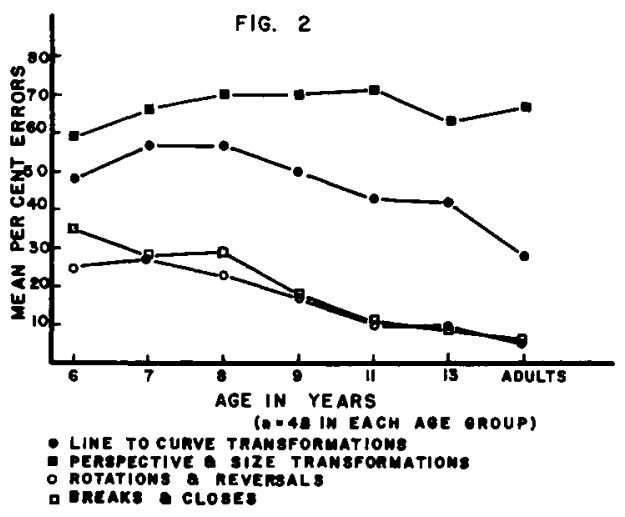

Fig. 2. Mean per cent discrimination errors for each type of transformation: normally sighted. 


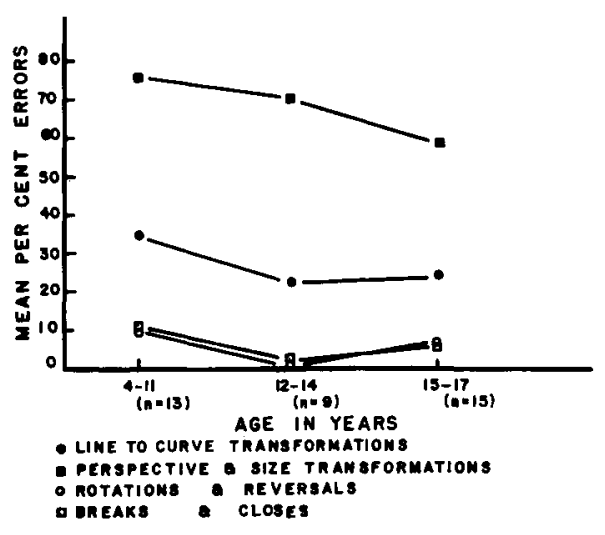

Fig. 3. Mean per cent discrimination errors for each type of transformation: partially sighted.

A supplementary group of 48 normally sighted adult college students was run under identical conditions. Their data were not included in the analysis of variance but as can be seen in Fig. 2, their results are continuous with those of the younger Ss.

Since there were so few blind Ss at any one age, the data were grouped according to three age groups. Again the suggestion in Figs. 3 and 4 of a difference in confusability of different transformation types was borm out by analysis of variance for partially sighted $(F=157.35, \mathrm{df}=3 / 102, \mathrm{p}<.01)$ and for totally blind $(\mathrm{F}=65.49, \mathrm{df}=3 / 102, \mathrm{p}<.01)$. In addition, there was a significant interaction between age and type of transformation for the totally blind $(F=2.53$, $d f=6 / 102$, $\mathrm{p}<.05)$.

\section{Discussion}

The decrease in errors as a function of age shown by the sighted Ss making tactual discriminations is, of course, an expected developmental trend and is similar to the trend found by Gibson et al (1962) with visual discrimination of these forms. Although differences in procedure preclude direct comparison with their data, it is quite obvious that the tactual discrimination of these forms is far more difficult than visual discrimination. The visual discrimination Ss were making practically no errors by 8 years of age whereas even the adults of the present study were making substantial numbers of errors.

The lack of improvement with age by both blind groups is somewhat surprising. It may be that for these particular stimulus dimensions such improvement requires normal vision and the improvement shown by the sighted Ss may be a case of intermodal transfer. If so, the present results provide a clue as to the kinds of stimulus dimensions that might be involved.

Of equal interest with the age trends is the relative difficulty of the types of transformation. In the visual discrimination study Gibson et al found the order to be: perspective most difficult, line to curve and rotations and reversals intermediate in difficulty, and break and close discriminations easiest. In the present study,

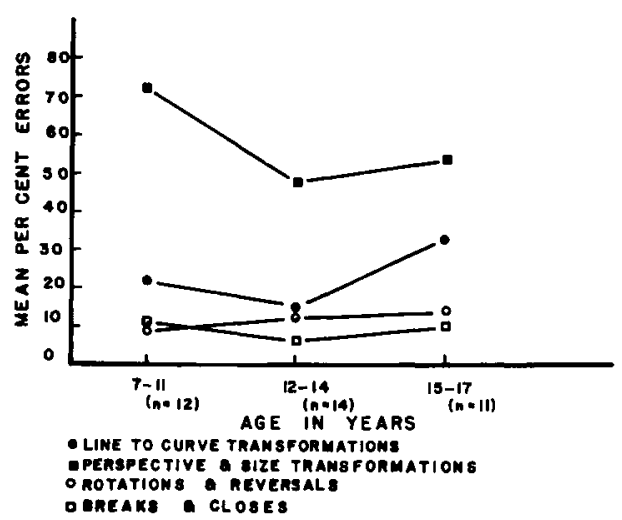

Fig. 4. Mean per cent discrimination errors for each type of transformation: totally blind.

perspective transformations were again found most difficult and breaks and closes were found very easy for all groups. However, rotations and reversals were found to be easy tactual discriminations for all the groups in contrast with the previous visual discrimination results. The ease of these tactual discriminations would seem. to be because an S, feeling corresponding locations on a standard stimulus and a rotation of it would feel a difference as great or greater than the difference between a standard form and a break or close transformation of it. The discrimination of line to curve transformations for the sighted Ss and for the partially sighted Ss are of intermediate difficulty in the present study in contrast to the totally blind Ss for whom this type of discrimination appears to be easier. The basis of this difference related to the amount of available vision is not clear.

The different types of transformation employed in the present study enable one to make qualitative statements about the relation between stimulus dimension discrimination and type of visual experience. However, the particular values of the stimulus dimensions are, to some extent, arbitrarily chosen and further research along this line should probably employ stimuli that vary more quantitatively along each stimulus dimension as well as varying across several stimulus dimensions.

\section{Reference}

Gibson, Eleanor J., Gibson, J. J., Pick, Anne D., \& Osser, H. A. A developmental study of the discrimination of letter-like forms. J. comp. physiol. Psychol., 1962, 55, 897-906.

\section{Note}

1. This research was supported by NIH Grant MH 07631 from the National Institutes of Health to the University of Minnesota. The cooperation of the officials, teachers, and students of the Faribault State School for the Blind, the Groveland Park and Ramsey public schools in St. Paul, Minnesota, and the Fall Creek public school in Ithaca, New York are appreciated. Drs. Robert Klein and Natalie E. Torrey, and Nancy Acker, Meridith Fink, James Hall, Gretchen Nadeau, Susan Roberts, Daniel Smothergill, and Margaret Thomas assisted in conducting various aspects of this study. 